\title{
IDENTIFICACIÓN Y ANÁLISIS DE LOS ERRORES DE MEDICACIÓN EN SEIS HOSPITALES BRASILEÑOS
}

\section{IDENTIFICATION AND ANALYSIS OF MEDICATION ERRORS IN SIX BRAZILIAN HOSPITAL}

\author{
Silvia Helena de Bortoli Cassiani* \\ Aline Aparecida Silvia MonZani ${ }^{* *}$ \\ Ana Elisa Bauer de Camargo Silva ${ }^{* * *}$ \\ Flávio Trevisani FaKiH ${ }^{* * * *}$ \\ Simone Perufo Opitz $Z^{* * * *}$ \\ Thalyta Cardoso Alux TeixeIra******
}

\begin{abstract}
RESUMEN
Esta investigación identificó, a través del método de observación directa, los errores de medicación ocurridos en unidades de clínica médica de seis hospitales brasileños. La muestra consistió de las dosis prescritas y administradas en las unidades y observadas durante el periodo de recolección de datos, obedeciendo a un mínimo de 35 dosis/día, durante 30 días. Los resultados evidenciaron los siguientes aspectos: el 1,7\% de los medicamentos administrados fue diferente de los prescritos; el 4,8\% de las dosis administradas difirieron de las prescritas; el $1,5 \%$ de los medicamentos fue administrado en vías diferentes de las prescritas; el $0,3 \%$ de los pacientes recibieron medicamentos no autorizados; cerca del 7,4\% de los medicamentos fue administrado más de $1 \mathrm{~h}$ después del horario previsto y el 2,2\% más de $1 \mathrm{~h}$ antes del prescrito.
\end{abstract}

Palabras clave: Errores de medicación, seguridad del paciente, administración de medicamentos.

\begin{abstract}
This study identified medication errors that occurred at the medical clinics of 6 Brazilian hospitals, using direct observation. The sample consisted of doses prescribed and administered at the clinics, which were observed during the data collection period, respecting a minimum of 35 doses/day, during a 30 -day period. The results evidenced that: $1.7 \%$ of the administered medication was different from the prescribed drugs; $4.8 \%$ of the administered doses were different from the prescriptions; $1.5 \%$ of the medication was administered through routes different from the prescriptions; $0.3 \%$ of patients received non-authorized medication; about $7.4 \%$ of the medication were administered more than $1 \mathrm{~h}$ after and $2.2 \%$ more than $1 \mathrm{~h}$ before the prescribed medication time.
\end{abstract}

Key words: Medication errors, patient safety, medication administration

Fecha recepción: 17/04/06 Fecha aceptación: 10/11/09

\footnotetext{
* Doctora. Enfermera. Docente de la Escuela de Enfermería de la Universidad de São Paulo - EERP-USP, Ribeirão Preto Brasil. E-mail: shbcassi@eerp.usp.br

** Mestre. Enfermera del Hospital Vera Cruz, Campinas. Docente de La Faculdade de Enfermería de La Pontifíca Universidade Católica - PUC - Brasil. E-mail: aline.monzani@uol.com.br

*** Doctora. Enfermera. Docente de la Facultad de Enfermería de la Universidad Federal de Goiás - UFG - Brasil. E-mail: anaelisa@terra.com.br

**** Enfermero del Hospital São Paulo - Universidad Federal de São Paulo - Brasil. E-mail: ffakih@unifesp.br

***** Doctora. Enfermera. Docente de la Universidad Federal de Acre - Brasil. E-mail: simoneopitz@hotmail.com

****** Mestre. Enfermera del Hospital Vera Cruz, Campinas. Doctoranda del Programa de Enfermería Fundamental de la EERP-USP - Brasil. E-mail: thalytacat@yahoo.com.br
} 


\section{INTRODUCCIÓN}

La publicación, en noviembre de 1999, del informe del Institute of Medicine (EEUU) intitulado To err is human: building a safer health system, que ha sido referencia para muchos estudios $(1,2)$, proveyó una gran propulsión a la preocupación con la seguridad del paciente. Este indicó que, en hospitales de EEUU, entre 44.000 y 98.000 americanos murieron debido a errores causados por profesionales de salud, que muchos de ellos se podrían haber prevenido y que excedieron las muertes por vehículos motorizados, cáncer de seno y AIDS (3).

Además de EEUU, varios otros países como Inglaterra, Irlanda, Australia, Canadá, España, Nueva Zelandia, Suecia y otros también han mirado con atención el tema de la seguridad de los pacientes y tomado iniciativas, tales como la creación de institutos, asociaciones y organizaciones. A título de ilustración, una consulta a la Base de Datos Medline en marzo de 2004 mostró que, en 2003, bajo el descriptor "errores de medicación" fueron publicados: 733 artículos en EEUU, 75 en Reino Unido, 70 en Canadá, 39 en Alemania, 36 en Francia y Dinamarca, 6 en México, 8 en Brasil y ninguno en los otros países de América Latina (4).

En 2002, la Organización Mundial de la Salud (OMS) recomendó, a través de la Resolución WHA 55.18, adoptar la seguridad del paciente como tema de alta prioridad en la agenda de políticas de los países miembros (5).

Brasil empezó a movilizarse hacia esa dirección en 1999, con la creación de la Agencia Nacional de Vigilancia Sanitaria por el Ministerio de la Salud. En 2001 se montó una red de 100 hospitales de alta complejidad, distribuidos en todo el territorio nacional, llamados "Hospitales Centinelas" que fueron motivados a notificar efectos adversos y quejas técnicas de productos de salud.

A partir del 2007, la Organización Pana- mericana de la Salud llevó a cabo reuniones con la participación de representantes de países de las Américas, tales como EEUU, Argentina, Chile, Brasil, entre otros, con objeto de consolidar un Grupo de Especialistas en el área de información y educación básica, continua y permanente en enfermería para promover la seguridad de los pacientes y proyectar un plan de trabajo en conjunto.

Estudios destacan que los hospitales deben focalizar sus esfuerzos y estrategias en el proceso de medicación, que representa la causa más común de eventos adversos, con un número considerable de personas afectadas, en el cual muchos daños son evitables, y para el cual ya existen muchos trabajos científicos y herramientas desarrolladas para prevención de errores $(2,6,7,8)$.

Un estudio destacó a los errores de omisión $(24 \%)$, de dosis o cantidad inadecuada (23\%) y los errores de prescripción (22\%) como los más frecuentes relacionados en MEDMARX, una base de datos, con acceso por internet, que recolecta datos anónimos y analiza errores de medicación, desarrollada por la United States Pharmacopeia (USP). Las principales causas asociadas a esos errores fueron el déficit en la realización de la actividad (38\%), no seguir procedimiento y protocolo $(20 \%)$ y transcripción inadecuada/omisión (15\%) (9).

Error de medicación, según el National Coordinating Council for Medication Error Reporting and Prevention (NCC MERP), es "cualquier evento evitable que puede estar relacionado a la práctica profesional, productos de cuidado de salud, procedimientos y sistemas, abarcando inclusive la prescripción, comunicación, rótulos, envase y nomenclatura, dispensación, distribución, administración, educación, monitoreo y uso" (10).

Son varios los factores que pueden llevar a errores en la medicación, abarcando desde la falta de atención, lapsos de memoria, deficiencias de la formación académica, inexperiencia, negligencia, hasta problemas en el sistema de medicación. 
Entre los investigadores existe un consenso de que el individuo raramente es la única causa, y que se debe considerar siempre las posibles fallas del sistema que pueden colaborar para la ocurrencia del error. Sin embargo, la identificación de errores de medicación es una primera medida para prevenirlos.

\section{OBJETIVO}

Este estudio identificó los errores de medicación ocurridos en unidades de clínica médica de 6 hospitales brasileños y detectados a través del método de observación directa.

\section{MATERIAL Y MÉTODOS}

Este estudio cuantitativo y descriptivo con diseño transversal fue realizado en unidades de clínica médica de seis hospitales, siendo cinco hospitales públicos y pertenecientes a la Red de Hospitales Centinela de la Agencia Nacional de Vigilancia Sanitaria y uno privado, no perteneciente a esta red.

Los hospitales centinela fueron seleccionados porque representan una estrategia para integrar el monitoreo de medicamentos a la práctica clínica en el país y también porque los autores de esa investigación actúan en estos hospitales.

La clínica médica fue elegida por ser una unidad de internación para pacientes con enfermedades crónico-degenerativas. Estas enfermedades generalmente requieren una gran cantidad de medicamentos durante el tratamiento de los pacientes, lo que lleva a la preparación y administración diaria de un gran volumen de dosis.

Esas instituciones son las siguientes: Hospital das Clínicas de la Universidad Federal de Goiás, Hospital das Clínicas de la Facultad de Medicina de Ribeirão Preto de la Universidad de São Paulo, Hospital São Paulo de la
Universidad Federal de São Paulo, Fundación Hospital Estadual de Acre, Hospital General de Fortaleza y Hospital Vera Cruz en Campinas - SP, que serán descritos como hospitales A, B, C, D, E y F, en orden diferente a la de esta descripción.

\section{Población y muestra}

El universo investigado fueron las dosis prescritas y administradas en las Unidades de Clínica Médica de los hospitales. Para determinarse el valor de la muestra, se calculó el número de medicamentos y dosis diarias administradas en las unidades. Se estableció que un $\mathrm{n}=35$ dosis/día sería observada en cada hospital.

Así, la muestra incluyó las dosis prescritas y administradas en las Unidades de Clínica Médica y observadas durante el periodo de recolecta de datos, al mínimo 35 dosis/día, durante 30 días.

\section{Aspectos éticos}

Tras la autorización y aprobación de los proyectos por los Comités de Ética en Investigación de cada una de las instituciones, se inició la recolección de datos.

\section{Capacitación, planificación del trabajo de campo e instrumento de recolección de da- tos}

Cada coordinador de la investigación en los hospitales fue un enfermero, miembro del equipo ejecutor y responsable por reclutar y capacitar a los observadores. El equipo seleccionó y contrató a tres observadores por hospital y planificó su capacitación, lo que exigió una carga horaria total de $20 \mathrm{~h}$.

\section{Desarrollo de los instrumentos de recolec- ción de datos}

Los instrumentos de recolección de datos fueron desarrollados específicamente para 
recolectar datos referentes a la preparación de la administración y prescripción de medicamentos. Esos instrumentos se basaron en un estudio anterior, realizado en 4 instituciones hospitalarias y fundamentado en datos de la literatura $(11,12,13)$, recolectando datos relacionados al ambiente, a los medicamentos y a la realización de los procedimientos. Fueron validados por cinco peritos, efectuando modificaciones según las sugerencias propuestas.

Luego se realizó el pretest en dos hospitales $y$, tras reuniones, se incorporaron ajustes en cuanto al cálculo muestral y a los ítemes de los instrumentos. Posteriormente, se llevó a cabo un estudio piloto en Ribeirão Preto, Goiânia y São Paulo.

El estudio piloto evidenció dificultades en la observación del número de dosis estipuladas, solucionadas por cambios con objeto de viabilizar la recolección, utilizando 3 observadores que trabajaron independientemente en la observación y el registro de dosis.

\section{Recolección y análisis de los datos}

Todos los miembros del equipo de enfermería (enfermeros, técnicos y auxiliares de enfermería) involucrados en el preparo y administración de los medicamentos en las unidades fueron invitados a participar de la investigación. Aquellos que aceptaron firmaron un término de consentimiento libre $y$ esclarecido.

Los datos fueron recolectados a través de la observación directa y no participante de la preparación y de la administración de los medicamentos realizados por los profesionales de enfermería. La técnica de observación directa ha sido descrita en la literatura como uno de los mejores métodos para detectar errores (1).

Se estableció la presencia de un observador en cada turno de trabajo de las instituciones y en los siguientes períodos: de las 08:00 a las 12:00, de las 14:00 a las 18:00 y de las 19:00 a las 00:00 $\mathrm{h}$, totalizando tres observadores/día, siendo 1 por período. El observador se aproximó a 1 de los profesionales de enfermería, identificándose y solicitando permiso para acompañarlo durante la preparación y la administración de los medicamentos. Tras el consentimiento, el observador iniciaba los apuntes en los instrumentos, registrando detalles del medicamento preparado y administrado. Después, proseguía con la anotación de las informaciones de la prescripción original de los pacientes medicados. Cada dosis observada era comparada con la prescrita. Si existía alguna diferencia entre las informaciones recogidas y aquéllas observadas en la prescripción, el error era identificado, descrito, categorizado e informado al profesional. Sin embargo, si una dosis había sido prescrita, pero no administrada, el error era definido como "error de omisión".

Por lo tanto, los observadores fueron considerados "ciegos", ya que no poseían conocimiento previo de la prescripción médica. También se determinó que los errores identificados durante la observación y que pudieran acarrear daños a los pacientes serían interceptados por los observadores.

Cada dosis observada en la preparación y la administración, además de aquéllas omitidas, fueron operacionalmente definidas como "una dosis", constituyéndose en un único dato observado.

Al término de la recolección, los datos fueron traspasados y analizados mediante EPIDATA versión 3.1 y SPSS 10.0 (SPSS Inc., Chicago, II., USA).

\section{RESULTADOS}

La Tabla 1 indica que 6.169 instrumentos fueron llenados de forma completa en las etapas de preparación, administración y prescripción. Esa cifra, en cada hospital, varió de acuerdo con el número de lechos y la configuración del proceso de preparación y 
administración de los medicamentos, en aspectos como: número de auxiliares de enfermería y su disposición para participar de la recolección de los datos, número de los me- dicamentos prescritos y administrados en el horario y capacidad de los observadores para registrar las situaciones.

Tabla 1: Distribución del número de instrumentos recolectados según los hospitales investigados. Ribeirão Preto, 2006.

\begin{tabular}{c|c|c}
\hline \multirow{2}{*}{ Hospital } & \multicolumn{2}{|c}{ Total de instrumentos llenados } \\
\cline { 2 - 3 } & $\mathbf{N}$ & $\mathbf{\%}$ \\
\hline A & 817 & 13,2 \\
$\mathbf{B}$ & 1.619 & 26,2 \\
$\mathbf{C}$ & 661 & 10,7 \\
$\mathbf{D}$ & 1.577 & 25,6 \\
E & 963 & 15,6 \\
F & 532 & 6,6 \\
\hline TOTAL & $\mathbf{6 . 1 6 9}$ & $\mathbf{1 0 0}$ \\
\hline
\end{tabular}

Los datos obtenidos fueron analizados, considerándose las incongruencias entre los datos recolectados a partir de la prescripción médica y aquéllos observados durante la administración de los medicamentos. La tabla 2 fue construida a partir del principio de los cinco correctos o exactos: el medicamento correcto o exacto en la dosis y vía correctas o exactos, administrado al paciente correcto o exacto, en el horario correcto o prescrito, y presenta un condensado de los datos presentados.

Tabla 2: Distribución de la frecuencia de error en la administración de los medicamentos por categoría y hospital estudiado. Ribeirão Preto, 2006.

\begin{tabular}{|c|c|c|c|c|c|c|c|c|c|c|c|c|c|c|}
\hline \multirow{3}{*}{ Categorías de error } & \multicolumn{12}{|c|}{ Hospitales } & \multirow{2}{*}{\multicolumn{2}{|c|}{ Total }} \\
\hline & \multicolumn{2}{|c|}{ A } & \multicolumn{2}{|c|}{ B } & \multicolumn{2}{|c|}{ C } & \multicolumn{2}{|c|}{ D } & \multicolumn{2}{|c|}{ E } & \multicolumn{2}{|c|}{$F$} & & \\
\hline & $\mathrm{N}$ & $\%$ & $\mathrm{~N}$ & $\%$ & $\mathrm{~N}$ & $\%$ & $\mathrm{~N}$ & $\%$ & $\mathrm{~N}$ & $\%$ & $\mathrm{~N}$ & $\%$ & $\mathrm{~N}$ & $\%$ \\
\hline $\begin{array}{l}\text { Medicamento no } \\
\text { autorizado }\end{array}$ & 7 & 0,9 & 6 & 0,4 & 8 & 1,2 & 71 & 4,5 & 8 & 0,8 & 3 & 0,6 & 103 & 9,8 \\
\hline Dosis equivocada & 21 & 2,6 & 20 & 1,4 & 23 & 3,7 & 169 & 11 & 41 & 4,7 & 3 & 0,6 & 277 & 26,4 \\
\hline Vía equivocada & 33 & 4,1 & 16 & 1,0 & 7 & 1,1 & 4 & 0,3 & 24 & 2,6 & 5 & 1,0 & 89 & 8,5 \\
\hline Horario equivocado & 28 & 5,0 & 201 & 35,6 & 128 & 22,7 & 113 & 20,0 & 90 & 1,6 & 4 & 0,7 & 564 & 53,8 \\
\hline Paciente equivocado & 2 & 0,3 & 5 & 0,3 & 2 & 0,3 & 2 & 0,1 & 3 & 0,3 & 2 & 0,4 & 16 & 1,5 \\
\hline TOTAL & 91 & 8,7 & 248 & 23,6 & 168 & 16,0 & 359 & 34,2 & 166 & 15,8 & 17 & 1,6 & 1.049 & 100 \\
\hline
\end{tabular}

Se observa que los errores relacionados al horario correspondieron al 53,8\% y los de dosis equivocada al $26,4 \%$, seguidos por medicamentos no autorizados $(9,8 \%)$, vía equivocada $(8,5 \%)$ y paciente equivocado $(1,5 \%)$. 
En cuanto a las diferencias entre los medicamentos prescritos y los administrados, indicativas de que el paciente no recibió el medicamento prescrito, se observaron índices entre el $0,4 \%$ y el $4,5 \%$ de errores en los hospitales, siendo el $0,9 \%$ en el hospital A, $0,4 \%$ en el hospital B, $1,2 \%$ en el hospital C, $4,5 \%$ en el hospital D, $0,8 \%$ en el hospital E y $0,6 \%$ en el hospital F. La suma de todos los medicamentos fue de 9,8\%, o sea, 98 en cada 1.000 medicamentos administrados difirieron de la prescripción.

El análisis de los datos del hospital $\mathrm{B}$, que mostró el menor valor, informa que, de las seis dosis administradas diferentes de las prescritas, cuatro estuvieron relacionadas a la falta del medicamento Berotec o Atrovent. Así, solamente uno de los dos fue administrado en la nebulización.

El hospital D presentó el mayor valor. Este fue debido a la omisión del medicamento Atrovent (que en el momento de la recolecta de datos no estaba disponible en la institución), cuando la prescripción indicaba asociación de Berotec y Atrovent. Se debe resaltar que, del total de 71 dosis incorrectas, 63 estaban relacionadas específicamente a la falta del medicamento Atrovent.

Respecto a las dosis en los hospitales A y $\mathrm{B}$, las dosis administradas diferentemente de las prescritas variaron entre el 2,6 y el 1,4\%. Entre las dosis consideradas incorrectas, hubo casos en que la prescripción determinaba, por ejemplo, una ampolla y el observador verificó que fue administrado $2 \mathrm{ml}$ de determinado medicamento, o entonces una medida y el observador verificó y anotó $5 \mathrm{ml}$.

Muchos errores en la dosis se refirieron a la administración de un comprimido en vez del medio comprimido o $1 / 4$ de comprimido prescrito. Ese dato fue notado principalmente en las observaciones del hospital A, en donde de 16 errores, 14 estuvieron relacionados a los comprimidos. Se puede afirmar, para todos los hospitales, que la mayoría de los errores de dosis se refirieron a los comprimidos, ocurriendo la administración de una superdosis (dos comprimidos en lugar de uno) o una subdosis (un comprimido en lugar de dos).

Para ilustrar, se observó una prescripción con Dexametasona - $4 \mathrm{mg}$. Sin embargo, la presentación fue de $5 \mathrm{mg} \mathrm{y}$, como consecuencia de la imposibilidad o dificultad en calcular la dosis exacta, fue administrado el comprimido entero de $5 \mathrm{mg}$, por lo tanto, incorrectamente. Ese dato indica que, muchas veces, en las prescripciones, la redacción de la dosis es como medida o ampolla y no con la dosis real del medicamento.

En el hospital B, los errores estuvieron relacionados a: prescrito Rocefin $2.000 \mathrm{mg}$ y administrado $1.000 \mathrm{mg}$, Tienam $500 \mathrm{mg}$ y administrado $1.000 \mathrm{mg}$, Solumedrol $20 \mathrm{mg}$ y ministrado $100 \mathrm{mg}$, como ejemplos. En el hospital C, los errores se refirieron a la administración de Amicacina: prescrito $1.000 \mathrm{mg}$, administrado $2.000 \mathrm{mg}$, Ácido Fólico, prescrito $2 \mathrm{mg}$ y administrado $5 \mathrm{mg}$, Flebocortid prescrito $200 \mathrm{mg}$ y administrado $500 \mathrm{mg}$ y Hidrocortizona $200 \mathrm{mg}$ y administrado 100 mg. También se observó una dosis incorrecta de insulina, cuando fueron administradas cuatro unidades en lugar de cinco.

El hospital D presentó el mayor número de casos en que fueron administradas dosis de medicamentos diferentes de las prescritas. Eso fue debido a la administración de 1.000 mg de Dipirona en lugar de 500 mg prescrito, Decadron, prescrito $4 \mathrm{mg}$ y administrado $10 \mathrm{mg}$ y errores en la redacción de la prescripción con dosificaciones inexistentes, tales como Cimetidina $20 \mathrm{mg}$ cuando el correcto es 200 mg y Penicilina Cristalina 400.000 UI, cuando el correcto es 4.000.000 UI.

Es importante enfatizar que, en algunos casos, el auxiliar de enfermería relató que las alteraciones en la prescripción de los medicamentos, principalmente en la dosis de la insulina, fueron efectuadas verbalmente por el médico, pero la prescripción no fue alterada.

En general y considerando el total de dosis, la Tabla 2 muestra que 277 , o sea el $4,8 \%$ 
de los medicamentos fue administrado en dosis diferentes de las prescritas. Entre las dosis totales se incluyeron las prescritas en mg y en otras formas farmacéuticas.

Este resultado muestra porcentaje inferior a aquél encontrado en una investigación de 36 instituciones hospitalarias americanas, en el cual se verificó que el 19\% de las dosis administradas estaba equivocado, siendo que las categorías más frecuentes de errores estaban relacionadas al horario equivocado (43\%), omisión de las dosis (30\%), dosis equivocada (17\%) y medicamento no prescrito $(4 \%)(13)$.

La Tabla 2 también demuestra que 89 dosis $(1,5 \%)$ fueron administradas en vías diferentes de las prescritas. Las observaciones indicaban situaciones en que el medicamento había sido prescrito en la forma de presentación inyectable, pero fue administrado en la presentación comprimido (cp) por vía oral o viceversa, $\mathrm{o}$ entonces cuando el medicamento fue administrado por sonda nasogástrica o entérica (SO), pero había sido prescrito por vía oral (VO) o viceversa.

En el Hospital A, la mayoría de las dosis incorrectas estaba relacionada al medicamento administrado por sonda nasogástrica o entérica (SO) cuando fue prescrita la administración por vía oral (VO), o viceversa. Varias cuestiones deben ser consideradas, tales como la administración concomitante de medicamentos orales y fórmulas enterales. La administración incorrecta de medicamentos puede resultar en tubos de alimentación obstruidos, disminución de la eficacia del medicamento, aumento de eventos adversos, incompatibilidades de la fórmula del medicamento, lo que causa perjuicios a los pacientes (14).

Muchas de esas situaciones ocurrieron cuando, por causa de la evolución del cuadro del paciente, la enfermería buscaba al médico para informar el estado del paciente y él, de forma verbal, autorizaba el cambio en la forma de presentación del medicamento y, consecuentemente, en la vía. Otras veces, cam- bios en la prescripción actualizada, cuando todavía se está siguiendo la prescripción del día anterior, pueden llevar a alteraciones. Sin embargo, se encontraron casos evidentes de administración de medicamentos en vías equivocadas.

Problemas de comunicación causan trastornos en las actividades del equipo multidisciplinar, a punto de que los profesionales se culpaban unos a otros por los errores, hechos que ocasionan desgastes emocionales, atrasos y/u omisión en la administración de los medicamentos, además de acarrear gastos innecesarios para las instituciones hospitalarias. Es necesario revisar los procesos de medicación, implementar acciones proactivas y estrategias, con objeto de mejorar la comunicación y garantizar una terapéutica medicamentosa eficiente y segura a los pacientes (15).

En cuanto a los errores relacionados a los pacientes, $16(0,3 \%)$ fueron identificados con relación a la administración de un medicamento no autorizado al paciente. Así, 3 pacientes de cada 1.000 internados en esos hospitales, de forma general, recibieron medicamentos no prescritos.

En este estudio, el instrumento solicitaba descripciones de situaciones en que se verificó la inadecuación o el error. La mayoría de las descripciones relacionadas a la preparación del medicamento indicaba las fallas técnicas, tales como: la falta del lavado de las manos; falta de desinfección de ampollas y frasco-ampollas y contaminación del émbolo de la jeringa; interrupciones y distracciones en la actividad; transcripción de la prescripción en etiquetas y cintas adhesivas que identificaban los medicamentos para administración, que también fueron utilizadas como rótulos, muchas veces incompletos, ocurriendo la falta principalmente del nombre del paciente y horario de la preparación. También se mencionó la preparación de medicamentos con anterioridad, habiendo casos de hasta una hora de antelación de preparación. Con relación al ambiente, se 
observó área insuficiente o inapropiada con ruidos e iluminación deficiente.

En cuanto a las descripciones de las inadecuaciones o errores durante la administración de los medicamentos, se encontraron resultados diferentes respecto a la falta del lavado de las manos, no utilización de guantes de procedimiento y administración de medicamento mientras realizaba concomitantemente una actividad con otro paciente. También se observaron los ruidos del ambiente y la falta de iluminación, principalmente por la noche (con un relato en que la iluminación del local era solamente la de la TV). Además, faltó orientación o explicación al paciente sobre el procedimiento o el medicamento. Fue notable la no utilización de la prescripción en la confirmación del nombre del medicamento, dosis y vía y del nombre del paciente en casi todos los hospitales. Se notó que el registro del medicamento administrado solamente ocurría en los finales del turno o en los horarios más tranquilos, y no inmediatamente tras la administración.

Con relación a los errores observados en la prescripción del medicamento, se observó principalmente el término de la prescripción manual de medicamentos en los casos de la prescripción electrónica, además de la ilegibilidad del nombre del médico que prescri- bió en la mayoría de las prescripciones del tipo manual. También se observaron prescripciones incompletas, con falta de presentación del medicamento, dosis y prescripción de nombres comerciales y genéricos. El uso de abreviaturas por los médicos fue constante en todos los hospitales, como por ejemplo HCTZ como hidroclorotiazida, IN como inhalación, ABD como agua bidestilada, U como unidades. Datos del Hospital D informaron que el $94,1 \%$ de las prescripciones contenía algún tipo de abreviatura.

En un estudio realizado en Argentina, el $92 \%$ de los entrevistados dijo que se involucraron alguna vez en errores de medicación notificados y uno de los factores asociados fue la ilegibilidad de la prescripción médica (16). Además de la ilegibilidad de éstas, prescripciones incompletas pueden causar errores de medicación. Las abreviaturas utilizadas, aunque economicen el tiempo del prescriptor, pueden causar equívocos de lectura o de interpretación, ya que una abreviatura puede tener más de un significado y también aumentar el trabajo de aquellos que intentan comprenderla(17).

La Tabla 3 presenta los datos relacionados a los horarios de la administración de los medicamentos, comparados a los horarios en las prescripciones médicas.

Tabla 3: Distribución de los horarios de los medicamentos administrados según los hospitales y el horario previsto en la prescripción médica, en las unidades de clínica médica de los hospitales estudiados* ${ }^{\star}$ Ribeirão Preto, 2006.

\begin{tabular}{|c|c|c|c|c|c|c|c|c|c|}
\hline \multirow[t]{2}{*}{ Hospitales } & \multicolumn{2}{|c|}{$\begin{array}{c}\text { Administrado hasta } \\
1 \mathrm{~h} \text { antes } \\
\text { del previsto }\end{array}$} & \multicolumn{2}{|c|}{$\begin{array}{l}\text { Administrado hasta } \\
1 \mathrm{~h} \text { después } \\
\text { del previsto }\end{array}$} & \multicolumn{2}{|c|}{$\begin{array}{c}\text { Administrado más de } 1 \\
\text { h después } \\
\text { del previsto }\end{array}$} & \multicolumn{2}{|c|}{$\begin{array}{c}\text { Administrado más de } \\
1 \mathrm{~h} \text { antes } \\
\text { del previsto }\end{array}$} & \multirow[t]{2}{*}{ Total } \\
\hline & $\mathbf{N}$ & $\%$ & $\mathrm{~N}$ & $\%$ & $\mathrm{~N}$ & $\%$ & $\mathbf{N}$ & $\%$ & \\
\hline A & 208 & 26,3 & 554 & 70,1 & 20 & 2,5 & 8 & 1,0 & 790 \\
\hline B & 97 & 6,2 & 1.274 & 81 & 156 & 9,9 & 45 & 2,9 & 1.572 \\
\hline $\mathrm{C}$ & 86 & 14,5 & 381 & 64 & 118 & 29,8 & 10 & 1,7 & 595 \\
\hline D & 675 & 43,2 & 766 & 49,6 & 63 & 4 & 50 & 3,2 & 1.564 \\
\hline E & 233 & 28,7 & 488 & 60,2 & 75 & 9,2 & 15 & 1,8 & 811 \\
\hline $\mathbf{F}$ & 19 & 3,7 & 502 & 95,4 & 3 & 0,6 & 1 & 0,3 & 525 \\
\hline TOTAL & 1.318 & 22,5 & 3.975 & 68,0 & 435 & 7,4 & 129 & 2,2 & 5.857 \\
\hline
\end{tabular}

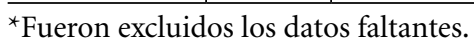


Se espera que el paciente reciba el medicamento prescrito en el horario previsto, siendo que la literatura indica un atraso o una anticipación de treinta minutos a una hora como posible. Sin embargo, la Tabla 3 muestra que cerca del $10 \%$ de los medicamentos fueron administrados antes o después del horario previsto. Este aspecto señala las fallas del sistema, generalmente relacionadas a la falta de personal o al número reducido de auxiliares o técnicos para prestar atención a los pacientes.

Se debe resaltar que, en la mayoría de los hospitales, los profesionales de enfermería son responsables por aproximadamente doce pacientes cada una, con niveles variados de complejidad. Ocurrió un caso en que, por la mañana, período éste con el mayor número de medicamentos prescritos, una única funcionaria del equipo de enfermería era responsable por la preparación y la administración de todos los medicamentos de cuatro enfermerías, las cuales contenían, en promedio, seis pacientes cada una. Así, se hace inviable la preparación y la administración de los medicamentos en los horarios prescritos.

Con relación al hospital, el mayor número de errores fue verificado en el hospital $\mathrm{D}$ $(34,2 \%)$, seguido por B $(23,6 \%), C$ (16\%), A $(8,7 \%)$ y $\mathrm{F}(1,6 \%)$. Es interesante la relación entre los errores y la presencia de prescripción médica electrónica, ya que el hospital A era el único que poseía este sistema. El hospital $\mathrm{F}$ era de tamaño medio y privado.

\section{DISCUSIÓN}

El proceso de administración de medicamentos en una organización hospitalaria puede ser visto como un sistema, siendo definido como una combinación de procesos interdependientes que comparten un objetivo común (18).

El sistema de medicación de un hospital es abierto y complejo, compuesto de varios procesos, desarrollando entre 60 y 70 pasos diferentes durante los procesos de prescripción, dispensación y administración de medicamentos, involucrando siempre a varios profesionales, lo que puede llevar a errores de medicación $(1,19)$.

El error puede ocurrir en cualquier momento, ya que el proceso de medicación ocurre en varias etapas secuenciales, dependientes una de otra y ejecutadas por un equipo multidisciplinar compuesto por médicos, farmacéuticos, enfermeras/ros y auxiliares. Por abarcar varias etapas e involucrar a varios profesionales, el riesgo de equivocarse es grande, y también la probabilidad de que un error potencial sea interceptado antes que afecte al paciente.

La Asociación Americana de Farmacéuticos Hospitalarios posee directivas para la prevención de errores de medicación en los hospitales, destinadas a pacientes, instituciones y profesionales. Algunas de esas medidas se refieren a la identificación del paciente en el hospital; verificación de la prescripción antes de la administración del medicamento al paciente; revisión de las prescripciones, verificando los resultados deseados para el paciente; duplicaciones terapéuticas y posibles interacciones medicamentosas $(2,9,20)$.

En este estudio fue recolectada una muestra de 6.169 dosis de medicamentos administrados a los pacientes internados en unidades de clínica médica de seis hospitales brasileños, evidenciando los siguientes aspectos:

a) el $1,7 \%$ de los medicamentos administrados fue diferente de los medicamentos prescritos, o sea, de cada 1.000 medicamentos administrados en las unidades de clínica médica investigadas, 17 eran diferentes de la prescripción médica.

b) el 3,3\% de las dosis administradas en mg y el $8,1 \%$ de las dosis prescritas en otras formas farmacológicas fueron diferentes de las 
prescritas. En suma, el 4,8\% de las dosis administradas fue diferente de las prescritas.

c) el 1,5\% de los medicamentos fue administrado en vías diferentes de las prescritas.

d) el $0,3 \%$ de los pacientes recibió medicamentos no autorizados o no prescritos.

e) el 2,2\% de los medicamentos fue administrado una hora antes del previsto y el 7,4\% más de una hora después del prescrito.

Resultados de otros estudios compatibles con éste indican valores muy diferentes entre varias investigaciones $(9,13,21,22)$.

En ninguno de los hospitales participantes existía un sistema de notificación de errores, lo que agrava el problema, pues pueden seguir ocurriendo, ya que no son detectados ni tampoco solucionados o prevenidos. Es importante facilitar la comunicación de los errores mediante instrumentos y registrar los cometidos de forma anónima para que no configure una búsqueda del culpado, pero sí aplicar la mejor manera de evitarlos. Inclusive en hospitales que utilizan informes de ocurrencias, se cree que exista la subnotificación debido al miedo de punición por parte de los profesionales (23, 24).

En Brasil, y también en Latinoamérica, las discusiones sobre esta temática se están apenas iniciando. Por lo tanto, no podemos ausentarnos de este escenario y dejar de contribuir, con objeto de auxiliar a los pacientes para encontrar mayor seguridad en sus tratamientos y ofrecer una atención segura y de calidad. También es imperativo, actual y primero, lidiar con la problemática de la identificación de errores de medicación entre los hospitales, a fin de proponer estrategias para su prevención. Nada mejor que evidenciar a través de las investigaciones lo que ya viene siendo observado en la práctica.

\section{LIMITACIONES DEL ESTUDIO}

Las mayores limitaciones de este estudio, como en otros trabajos encontrados en la literatura, estuvieron relacionadas al uso de la técnica de observación para recolectar los datos. Esas se refirieron tanto a dificultades para que el equipo de enfermería aceptara ser observado, como a dificultades del propio observador, que puede haber interferido en la situación, simplemente debido a su presencia (Efecto Hawthorne). Esa última limitación fue prevenida a través de la presencia del equipo de observadores por un período de 30 días.

Otra limitación estuvo relacionada al miedo permanente de sanciones entre los profesionales de enfermería cuando son descubiertos errores o fallas técnicas, además del recelo de los jefes en revelar datos que reflejan la realidad de trabajo y las condiciones de los hospitales. La relación entre errores de medicación y ambientes punitivos contribuyó para algunas dificultades encontradas en la aceptación del equipo de observadores.

\section{REFERENCIAS}

1. Flynn E, Barker KN, Pepper G, Bates DW, Mikeal RL. Comparison of methods for detecting medication error in 36 hospitals and skilled-nursing facilities. Am J Health Syst Pharm. 2002; 59:436-46.

2. Miasso AI, Cassiani SHB. Administração de medicamentos: orientação final de enfermagem para a alta hospitalar. Rev Esc Enferm USP. 2005; 39(2):136-44.

3. Kohn LT, Corrigan JM, Donaldson MS. Committee on Quality Health Care in America, Institute of Medicine. To err is human: Building a safer health system. Washington (DC): National Academies Press; 2000. 
4. Cassiani SHB. A segurança do paciente e o paradoxo no uso de medicamentos. Rev Bras Enferm. 2005; 58(1):95-9.

5. Organização Mundial da Saúde - OMS. Declaration Pharmacists and public health. Hallado en: http://www.euro.who. int/document/epf/HelsinkiDecl2003.pdf [Acceso el 30 mayo de 2004].

6. Bohomol E. Erros de medicação: causas e fatores desencadeantes sob a ótica da equipe de enfermagem [disertación de maestría]. São Paulo (SP). Escola Paulista de Medicina da Universidade Federal de São Paulo; 2002. 143p.

7. Lee P. Ideal principles and characteristics of a fail-safe medication-use system. Am J Health Syst Pharm. 2002; 59(4):369-71.

8. Womer RB, et al. Multidisciplinary systems approach to chemotherapy and holding the gains. J Clin Oncol. 2002; 20(24):4705-12.

9. Santell JP, Hicks RW, McMeekin J. Medication errors: experience of United States Pharmacopea (USP) MEDMARX reporting system. J Clin Pharmacol. 2003; 43:760-67.

10. National coordinating council for medication error reporting and prevention NCC MERP. Taxonomy of medication errors. NCC MERP. Hallado en: http:// www.nccmerp.org/public/aboutmederror.html [Acceso el 09 septiembre de 2009].

11. Hatfield G. Rating hospitals for medication safety: a scorecard. Pharm Times. 1999; 65(9):42-5.

12. Telles Filho PCP, Cassiani SHB. Administração de medicamentos: aquisição de conhecimentos e habilidades requeridas por um grupo de enfermeiros. Rev Latino-Am Enferm. 2004; 12(3):533-40.

13. Barker KN, Flynn E, Pepper G, Bates DW, Mikeal RL. Medication errors observed in 36 health care facilities. Arch Intern Med. 2002;162(16): 1987-903.

14. Willians NT. Medication administration through enteral feeding tubes. Am J
Health Syst Pharm. 2008; 65(24):2347-57.

15. Silva AEBC, Cassiani SHB, Miasso AI, Optiz SP. Problemas na comunicação: uma possível causa de erros de medicação. Acta Paul Enferm. 2007; 20(3):272-6.

16. Cometto MC, Gómez P. Error en la administración de medicamentos en Enfermería. Notas enferm. 2008; 08(12):29-32.

17. Morais, J. A medicina doente. Isto é, SP. 2001; 15(5):48-58.

18. Nadzan DM. A system approach to medication use. In: Cousins DM. Medication use: a system approach to reducing errors. Oakbrook Terrace; 1998.

19. Leape LL, et al. Reducing adverse drug events: lessons from a breakthroughs series collaborative. Jt Comm J Qual Improv. 2000; 26(6):321-31.

20. Cohen MR. Medication errors: causes, prevention, and risk management. Washington (DC): Jones and Bartlett Publishers; 2000.

21. Schneider MP, Cotting J, Pannatier A. Evaluation of nurses' errors associated in the preparation and administration of medication in a pediatric intensive care unit. Pharm World Sci. 1998; 20(4):17882.

22. Koop BJ, Erstad BL, Allen ME, Theodorou AA, Priestley G. Medication errors and adverse drug events in an intensive care unit: direct observation approach for detection. Crit Care Med. 2006; 34(2):415-25.

23. Pepper G. Pesquisas em segurança na administração de medicamentos. In Cassiani SHB, Ueta J (Ed.). A segurança dos pacientes na utilização da medicação. São Paulo, SP: Artes Médicas; 2004. p. 93-109.

24. Monzani AAS. A ponta do iceberg: o método de notificação de erros de medicação em um hospital geral privado no município de Campinas-SP [dissertação de mestrado]. Ribeirão Preto (SP). Escola de Enfermagem de Ribeirão Preto da Universidade de São Paulo; 2006. 120p. 